\title{
Biodegradable, Biocompatible, Bioinspired and Bioabsorbale (Edible) Functional Materials for Solar Cell Applications
}

\section{Kumar CV* and Baveghems C}

*Department of Chemistry, University of Connecticut, Storrs CT 06278, USA

We, as a society, are facing the greatest challenges of all times, climate change, energy crisis, pollution etc., all at once. Providing affordable, sustainable and green technologies are essential but we need to minimize human impact on our environment by creating biodegradable functional materials. Nature is the master of the materials world: It makes green, sustainable, biodegradable, biocompatible, bioabsorbable, recyclable, edible functional materials. The green leaf is an edible solar cell, the brain is a biodegradable computer, birds are biodegradable flying machines, eyes are biodegradable high-resolution cameras etc. Only hundred years ago our major means of personal transportation was a horse buggy, which is completely biodegradable, bioabsorbable, green, and sustainable, including the driver and the motor (horse). The present day car is an excellent vehicle but a lot of that is made of materials that do not biodegrade, some of it not recycled and accumulates in the environment.

Inspired by nature, our group and collaborators are striving to make biological materials that fit these stringent conditions of being bio-friendly and edible to some organisms, plants or animals, while maintaining full functionality. As chemists we can address this issue and there are great challenges to overcome. How to make edible solar cells or iPads that can be composted? Our long-term goal is to make a bio-solar cell made from biodegradable components, which will use sunlight but produce electrical current or a liquid fuel for later use, while consuming carbon dioxide. This is an enormous challenge, and there are no examples of such bio-solar cells to the best of our knowledge, other than the natural system. The futuristic bio-solar cells will biodegrade at the end of their life, not during the use and should be compostable and not pollute the environment. Thus, both the energy crisis and climate change can be addressed simultaneously but the road to this goal is very long and very challenging as well as exciting. With adequate funding from federal agencies and industry, and support from other chemists, this goal can be achieved for the greater good of our community and longer-term viability of human habitability of our planet.

The concept of the artificial leaf (bio-solar cell) could consist of a self-assembled light harvesting system that is completely compostable and biodegradable [1]. Our group has developed a strategy (Schemes 1) that facilitates energy transfer cascade and downshifts high-energy UV photons in the blue region to red where solar cell function is better. The system is easily self-assembled using biodegradable components such as DNA, protein and dyes (Schemes 1). These components form large, highly ordered structures, as shown, on millimeter length scales. This self-assembly positions the dyes with optimal Forster radius thus facilitating highly efficient energy transfer from the initial donor dye (Hoechst 33258) to the terminal acceptor (Rhodamine B) where the accumulated photon energy could be harvested to drive solar cells or fuel production. The intermediary jumper dyes (coumarin and fluorescein) were strategically placed in the energy cascade: Coumarin 540A absorbance spectrum overlaps with Hoechst emission, fluorescein absorbance overlaps with the Coumarin 540A emission, and Rhodamine B absorbance overlaps with fluorescein emission. Excitation of the blue absorbing dye at $350 \mathrm{~nm}$ resulted in intense red emission at $600 \mathrm{~nm}$ with an enhancement factor of 2.4.

Such cascade dye sensitized solar systems may provide novel biological materials platforms required for biodegradable, compostable devices. In fact, this system is modular such that particular dyes could be omitted or the cascade could be extended by the addition of additional dyes that emit at progressively longer wavelengths. Since several of the commercial solar cells are more efficient in the red than at the blue wavelengths, this strategy of spectral shifting could be profitable to improve solar cell efficiency. Previous light harvesting systems from our laboratory used inorganic matrices [2,3] Moving forward toward a greener solar cell, we reported earlier a biodegradable light harvesting system [4] but it was solution based and it did not emit in the red region. Energy transfer from the DNA bases to a red-shifting was constructed by us [5] but a single chromophore was used which lacks the cascade effect required to harvest light over a wide wavelength range of the solar spectrum. Therefore, solid-state films of the above system shown in Schemes 1 are more suitable for solar applications. Still many hurdles must be passed for a practical operational device with reasonable usage life at an affordable price.

In summary, with the world's population increasing and energy resources dwindling amidst increased global warming brought on by pollution, a clean, green, low cost, biodegradable, and compostable form of solar cell is urgently needed within the near future to save the human habitat on this planet. President Obama's Clean Power act is the strong political support for this direction of work. The science community can make this a successful policy by vigorously contributing to this goal. According to the US Department of Energy [6] much progress has been made in the development of clean coal, low cost photovoltaic cells, and record solar cell conversion efficiencies of 43.5\%. However, solar cells that are highly efficient, clean, green, low cost, compostable, or biodegradable are urgently needed.

In the Schemes 1 above, some details are pertinent for discussion. The serum protein, bovine serum albumin is negatively charged and has no interest in self-assembling with DNA. So our group has amidated its $\mathrm{COOH}$ groups with the amine functions of triethylenetetramine such that the chemically modified protein is now highly positively charged $(+16)$. And this charge reversal of the protein has facilitated the strong association of the modified albumin with the negatively charged DNA

*Corresponding author: Kumar CV, Department of Chemistry, University of Connecticut, Storrs CT 06278, USA, E-mail: challavijayakumar@gmail.com

Received September 04, 2015; Accepted September 05, 2015; Published September 12, 2015

Citation: Kumar CV, Baveghems C (2015) Biodegradable, Biocompatible Bioinspired and Bioabsorbale (Edible) Functional Materials for Solar Cell Applications. Chem Sci J 6: 106. doi:10.4172/2150-3494.1000106

Copyright: ( $) 2015$ Kumar CV, et al. This is an open-access article distributed unde the terms of the Creative Commons Attribution License, which permits unrestricted use, distribution, and reproduction in any medium, provided the original author and source are credited. 


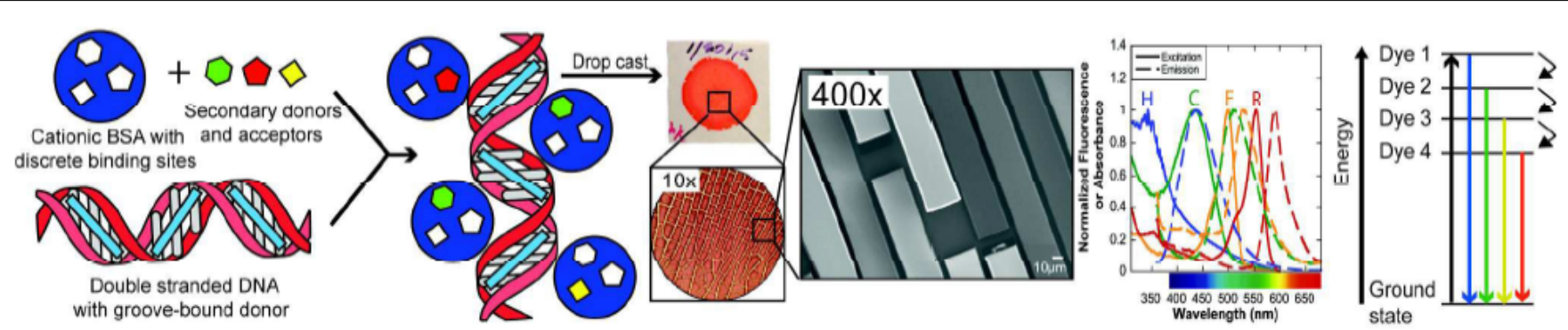

Scheme 1: Light harvesting by exciting Hoechst 33258 at $350 \mathrm{~nm}$. Light is red shifted from the primary donor to the terminal acceptor (Rhodamine B). Hoechst 33258 binds DNA while Coumarin 540A, Fluorescein, and Rhodamine B are embedded in cationized BSA (blue circles). The entire assembly is drop casted and they naturally formed long tapes of highly ordered antennas. Excitation at $350 \mathrm{~nm}$ of these films produced intense emission at $600 \mathrm{~nm}$ with an increase in the red emission by a facotr of 2.4 when compared to emission from the Rhodamine B alone, under otherwise identical conditions.

to form the protein-DNA matrix in the solid state. Previous work from our group showed that a number of organic molecules bind either to the DNA or to the albumin, and we exploited these studies to insert the above dye molecules into specific locations in the protein-DNA matrix. This position of dyes at particular sites is the key.

Our biodegradable antenna system is attractive for solar applications because the antenna is made from cheap biological/organic materials and is self-assembled easily and quickly, rendering it environmentally friendly and cost effective [7]. Thermal cycling studies where our antenna films are heated to $80^{\circ} \mathrm{C}$ and cooled back to room temperature withstood nearly 250 days of heating, and resisted degradation by heat. Thus, new benchmarks are set for the stability, biodegradability and function of antenna systems and these are currently under evaluation by commercial interests. The challenge will be to efficiently couple our edible light harvesting antenna with appropriate solar cells for enhanced electrical output while keeping the costs down.

\section{References}

1. https://youtu.be/qWLjMzl8u_Y?list=PLLG7h7fPoH8IObHbe9mnjum8w hIEXQnH>https://youtu.be/qWLjMzl8u_Y?list=PLLG7h7fPoH8IObHbe9mnjum $8 \mathrm{w} \_$hIEXQnH

2. Kumar CV, Novak MJ, Benson KR, Baveghems C, Thilakarathne VK, et al (2015) Toward the design of bio-solar cells: high efficiency cascade energy transfer among four donor-acceptor dyes self-assembled in a highly ordered protein-DNA matrix. RSC Advances 5: 72416-72422.

3. Kumar C, Chaudhari A, Rosenthal G (1994) Enhanced Energy Transfer between Aromatic Chromophores Bound to Hydrophobically Modified Layered Zirconium Phosphate Suspensions. J Am Chem Soc 116: 403-404.

4. Kumar CV, Chaudhari A (2000) Probing the Donor and Acceptor Dye Assemblies at the Galleries of $\alpha$-Zirconium Phosphate. Microporous and mesoporous materials 41: 307-318.

5. Duff MR Jr, Kumar CV (2009) DNA-Based Supramolecular Artificial Light Harvesting Complexes. J Am Chem Soc 131: 16024-6026.

6. Kumar C, Asuncion EH, (1993) DNA Binding Studies and Site Selective Fluorescence Sensitization of an Anthryl Probe. J Am Chem Soc 115: 85478553.

7. Herb J (2012) NREL Subcontract Report. NREL/SR-5200-54721. 